\title{
A role for the paravermis in the control of verbal interference: comparison of bilingual and monolingual adults
}

\author{
Roberto Filippi $^{1,2}$, Eva Periche-Tomas ${ }^{1,2}$, Andriani Papageorgiou ${ }^{1,2}$, and Peter Bright ${ }^{2,3}$ \\ ${ }^{1}$ University College London, Institute of Education, London WC1H 0AA \\ 2. MULTAC (Multilanguage and Cognition Lab), UCL Institute of Education, London WC1H 0AA \\ ${ }^{3}$ Anglia Ruskin University, Cambridge, CB1 1PT
}

\section{Corresponding author:}

Roberto Filippi

University College London - Institute of Education

Department of Psychology and Human Development

25, Woburn Square

London - WC1H 0AA

United Kindgdom

\section{Contact details:}

r.filippi@ucl.ac.uk

Acknowledgements: This work was supported by the Leverhulme Trust UK [RPG-2015-

024]. We thank Prof. Cathy Price for her valuable comments. A special thought goes to Prof. Annette Karmiloff-Smith who inspired our research. 


\begin{abstract}
We evaluate brain structure sensitivity to verbal interference in a sentence interpretation task, building on previously reported evidence that those with better control of verbal interference show higher grey matter density in the posterior paravermis of the right cerebellum (Filippi et al., 2011). We compare brain structure sensitivity to verbal interference control across two groups, English monolingual $(\mathrm{N}=41)$ and multilingual $(\mathrm{N}=46)$ adults. Using voxel-based morphometry, our primary goal was to identify and explore differences in regional patterns of grey matter sensitivity to performance on the sentence interpretation task, controlling for group variability in age, nonverbal reasoning and vocabulary knowledge. There was no group difference in performance but there was a significant group effect in grey matter sensitivity to task performance in our region of interest: stronger sensitivity in the paravermis in bilinguals compared to monolinguals in accuracy performance in the high (relative to low) verbal interference condition. This effect was observed when the linguistic interference was presented in an unfamiliar language (Greek) but not when presented in the familiar language (English). Our findings suggest that multilanguage acquisition mediates regional involvement within the language network, conferring enhanced functional plasticity within structures (including the paravermis) in the service of control of linguistic interference.
\end{abstract}




\section{Introduction}

Auditory control of interference is the cognitive ability to inhibit the processing of concurrent irrelevant auditory stimuli. This ability could be particularly beneficial for people who have to monitor, process and communicate in more than one language (i.e., the bilingual population). The aim of the current study was to use MRI structural imaging to investigate the neural correlates of verbal interference during speech comprehension in monolingual and bilingual populations, with specific focus on the cerebellum. Previous studies looking into the control of verbal interference have highlighted the involvement of the left caudate during an inhibitory control task based on colour naming (Ali et al., 2010) and left inferior or middle frontal regions when bilinguals were making decisions on a semantic written task in their native language (Rodriguez-Fornells et al., 2002, 2005). Until recently, the literature has been relatively silent on the candidate role of the cerebellum in this function, perhaps due in part to the tendency in many functional MRI studies to remove the cerebellum in its entirety prior to statistical analysis. However, over the past decade or so functional, lesion and developmental studies have shown and highlighted the importance of this structure in language processing (Jansen et al., 2005; Schweizer et al., 2010; Alexander et al., 2007; Krienen and Bucker, 2009; Hodge et al., 2010)

A specific area in the cerebellum, the most medial part of lobule VIIIA that lies within the posterior paravermis has been associated with the control of motor movements (Stoodley and Schmahmann, 2009). Furthermore, a more recent study has shown that the right posterior paravermis is involved in the control of verbal interference (Filippi et al., 2011) indicating a role for this structure in both motor and language functions. These authors employed structural neuroimaging in a sample of Italian bilingual participants (mean age 33 years old) who were late English learners, to identify brain regions that were positively correlated with 
the ability to control verbal interference. This was achieved through the administration of a dichotic listening task in which the participants were required to identify the agent in a series of sentences that differed in terms of structural complexity and in the absence and/or presence of interfering sentences. Results indicated that a cerebellar area, the right posterior paravermis, had higher grey matter density in those who were better at controlling verbal interference. These findings were consistent with a functional imaging study which investigated areas of activation when bilingual German participants (age range 23-62), who had been speaking English for more than 4 years, completed a semantic decision task while reading a list of words in the presence of distracting written stimuli (Filippi et al., 2011).

The current study followed the procedures implemented by Filippi et al. (2011), building on the experimental tasks and the target population. The sample in this study included monolingual and bilingual adults to explore any potential differences within the two language groups. In addition, the bilingual sample included a broad range of language backgrounds and experience. This study further modified the original experimental task by increasing the upper age threshold from approximately 40 to 80 years old.

\section{Methods}

\section{Participants}

The study included 87 right-handed adults between 18 and 80 years old (48 females, mean age 45.9 $\mathrm{SD}=19.3$ ) of which 41 were monolinguals and 46 were bilinguals. All participants spoke English and were residents in the UK. The bilingual sample represented a wide range of English language abilities. The study was approved by the local ethics committee and all participants gave written informed consent. 


\section{Materials and procedure}

Participants completed a background online questionnaire (Filippi et al., 2012, 2015) and were pre-screened for MRI safety before attending the testing sessions. Eligible participants were invited to complete a behavioural and structural imaging session which took place at UCL. The behavioural procedure included a battery of 4 tasks that measured vocabulary knowledge in English language (BPVS III), non-verbal reasoning (Raven's), verbal working memory (Digit span) and an auditory interference task (Sentence interpretation task). All information and instructions were given in English.

\section{BPVS III (British picture vocabulary scale III)}

This task uses 14 sets of 12 slides (Dunn and Dunn, 1997). Each slide presents four pictures (1 target and 3 distracters) with an auditory cue corresponding to the target image. All participants started from set 10, designated for ages of 14 and above and progressed to the next set only if all items where correct. One or more errors in set 10 results in moving to set 9 and this rule applies until all items within a set are correct. The task was completed if they reached set 14 or if they made 8 mistakes in a particular set. The final score was computed by subtracting the number of errors from the highest possible score.

\section{Raven's progressive matrices task}

This test of non-verbal fluid intelligence includes 12 increasingly complex trials (Raven, Raven and Court, 1998) with an estimated completion time of ten minutes. Each trial involves identifying the missing part from a geometric shape. Participants were required to select the correct part from eight candidate choices. One point is awarded for each correct answer.

\section{Digit span}


Participants are instructed to listen to a sequence of numerical digits (Wechsler, 2008). The aim is to repeat the sequence verbatim or in reverse order as instructed by the researcher. Participants were presented with two sequences of two digits. If at least one sequence was answered correctly the next set of sequences including one additional digit was subsequently presented. This was repeated until either two consecutive mistakes for a given sequence length were made or a sequence with nine digits was reached. This process was followed first for the forwards and then for the backwards condition. Scores were recorded according to the number of sequences answered correctly.

\section{Sentence Interpretation task}

The sentence interpretation task (Filippi et al., 2012, 2015) was programmed and conducted using E-Prime (version 2.0; Schneider et al., 2002). This study extended Filippi et al.'s (2012, 2015) sentence interpretation task to the comprehension of English target sentences in the presence of competing speech in English or in Greek, a language that was not known by any participant. Each sentence featured two animals and participants were asked to determine which of the two animals was performing a bad action towards the other (e.g., biting, bumping, pushing). For example, in the sentence the rabbit is scared by the horse, the horse is the bad animal. The animals featured in the target sentence simultaneously appeared on the screen, one animal on the left and one on the right; the participant pressed the corresponding left/right button on the gamepad to indicate which animal was being bad (see Figure 1).

The target sentences varied in their syntactic complexity, which altered their cognitive load (Roland, Dick and Elman, 2007). The easy canonical sentences (Subject-Verb-Object: $\mathrm{S}-\mathrm{V}-\mathrm{O}$ ) are less cognitively demanding than the hard non-canonical sentences (Object-VerbSubject: O-V-S or Object-Subject-Verb: O-S-V; Roland et al., 2007). Each of the English target sentences were presented binaurally with one of three levels of audible interference: (1) no interference - control condition, (2) English speaking interference, (3) Greek speaking 
interference. Competing sentences in English and Greek always featured a different animal and had different grammatical constructions. A pseudorandom pairing of target and interference sentences ensured that there was no overlap within trials between target animals and actions and interfering animals and actions.

Participants were provided with instructions that included listening to the sentence first and then choosing the bad animal. A practice run was first presented which included two blocks, one with a male and the other one with a female voice, uttering the target sentence. Participants were instructed to listen only to the voice stated at the beginning of each block and ignore the voice of the opposite gender.

Block presentation for the two practice blocks and two experimental blocks were counterbalanced so that half of the participants had a male target voice for the first block and female for the second block and vice versa). The practice blocks included eight trials each and the experimental block included 144 trials. For each trial the animal stimuli appeared on the screen for a maximum of $3000 \mathrm{~ms}$ or until a response was selected.

Visual stimuli consisted of black and white animal drawings on a grey background $(70 \mathrm{~mm} \mathrm{x}$ $50 \mathrm{~mm}$ ) taken from picture databases (Abbate and LaChappelle, 1984; Snodgrass and Vanderwart, 1980). Verbal auditory stimuli were 144 target sentences spoken by an English female, 144 target sentences spoken by an English male, 24 English interference sentences spoken by an English female and 24 English interference sentences spoken by an English male, 24 Greek interference sentences spoken by a Greek female and 24 Greek interference sentences spoken by a Greek male. Trials were presented in a random order. 


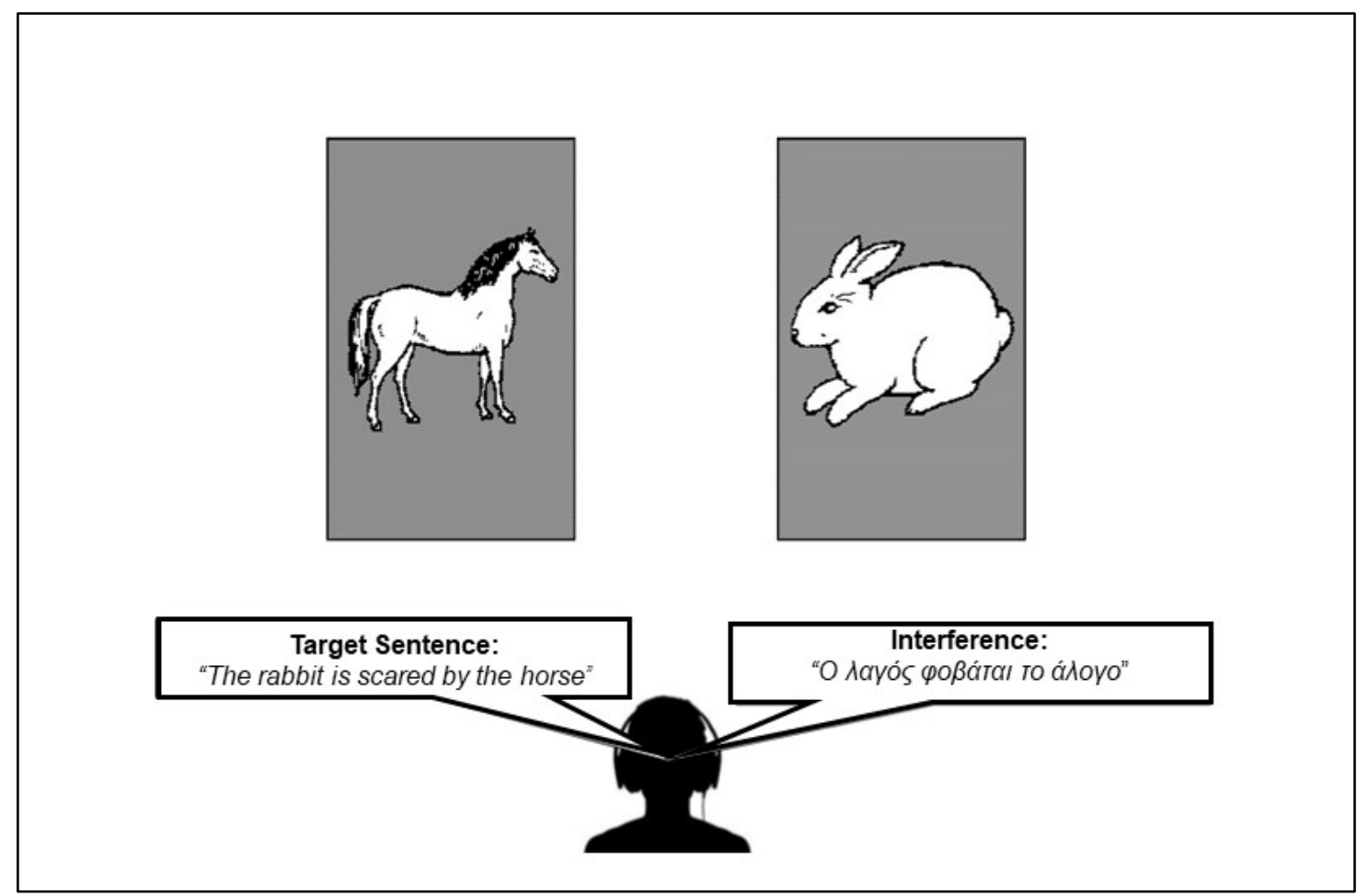

Figure 1: An illustration of the sentence interpretation task. Participants were instructed to indicate the animal doing the bad action as fast and accurately as possible. Target sentences were always in English. Competing sentences were either in English or in Greek and always spoken by the opposite gender (e.g., target: man's voice, interference: woman's voice or viceversa). Control trials were administered without verbal interference.

\section{Structural Image acquisition}

Whole brain anatomical T1-weighted images were acquired on a Siemens Sonata scanner housed in the Birkbeck-UCL Centre for Neuroimaging (BUCNI). For each of the 87 participants, 176 sagittal slices were collected with 256 × 224mm image matrix, providing a $1 \mathrm{~mm}^{3}$ voxel resolution $(\mathrm{TR} / \mathrm{TE} / \mathrm{TI}=12.24 / 3.56 / 530 \mathrm{~ms})$. 


\section{Structural Image analysis}

Pre-processing was applied using the Computational Anatomy Toolbox (cat12) for SPM12 (http://www.neuro.uni-jena.de/cat/). After manual inspection to ensure approximate alignment, initial segmentation was applied using the SPM tissue probability maps and subsequently the tissue probability atlases provided by the International Consortium for Brain Mapping (ICBM). Spatial registration was undertaken in MNI space using a DARTEL template derived from 555 healthy control subjects in the IXI-database (https://braindevelopment.org/ixi-dataset/) and the shooting template supplied as part of the cat12 toolbox. In order to correct for volumetric changes associated with nonlinear spatial normalisation, voxel values in the segmented grey matter were multiplied by the Jacobian determinant (volume changes) derived from the spatial normalisation stage. These modulated images, therefore, retained the total amount of grey matter from the original images, thereby providing the basis for analysis of volumetric differences between monolingual and bilingual brains and detection of regional volume sensitivity to behavioural performance on our tasks. Images were normalised to Montreal Neurological Institute (MNI) stereotaxic space, and smoothed using an isotropic kernel of $8 \mathrm{~mm}$ at full-width half-maximum (FWHM).

\section{Statistical analysis of structural data}

A full factorial interaction model was built to identify group effects (ML vs BL) while factoring out variance associated with i. age, ii. Raven's Matrices raw score, iii. BPVS III scores. Mean task scores on the non-canonical (i.e., 'difficult'), canonical ('easy') interference and control trials in the sentence interpretation task were entered as covariates separately modelled for each group (ML, BL). Scores in both English interference and Greek interference conditions were included in the model. We used volumes of interest within the cerebellum based on findings reported in Filippi et al. (2011), as described below. 


\section{RESULTS}

\section{Behavioural analyses}

Accuracy scores in the control condition (i.e., without language interference) were subtracted from those in the verbal interference conditions to obtain a task ability score. The scores are on a negative scale because performance on non-interference (baseline) tasks was generally better than that of interference conditions. As a result, a less negative score indicates better ability to manage interference. All analyses were controlled for age, English vocabulary knowledge, working memory and non-verbal reasoning ability scores. For control of interference, participants had worse performance when the task involved non-canonical sentences with English interference, the known language (Fig. 2).

This was confirmed by a $2 * 2 * 2$ mixed ANOVA on errors, showing a main effect of interference (English, Greek), $F(1,85) 72.12, p<0.001, \eta_{\mathrm{p}}{ }^{2}=.46$, and a main effect of sentence type (canonical, non-canonical), $F(1,85) 13.52, p=0.001, \eta_{\mathrm{p}}{ }^{2}=.14$, but no significant main effect of group (bilinguals, monolinguals), $F(1,85) .91, p=0.34, \eta_{\mathrm{p}}{ }^{2}=.01$, and no significant group* sentence and group*interference interaction, $F(1,85) 2.85, p=$ $0.10, \eta_{\mathrm{p}}{ }^{2}=.03$; interaction, $F(1,85) .68, p=0.78, \eta_{\mathrm{p}}{ }^{2}=.46$, respectively. The three-way interaction sentence*interference* group was also non-significant, $F(1,85) .71, p=0.40==, \eta_{\mathrm{p}}{ }^{2}$ $=.01$. 


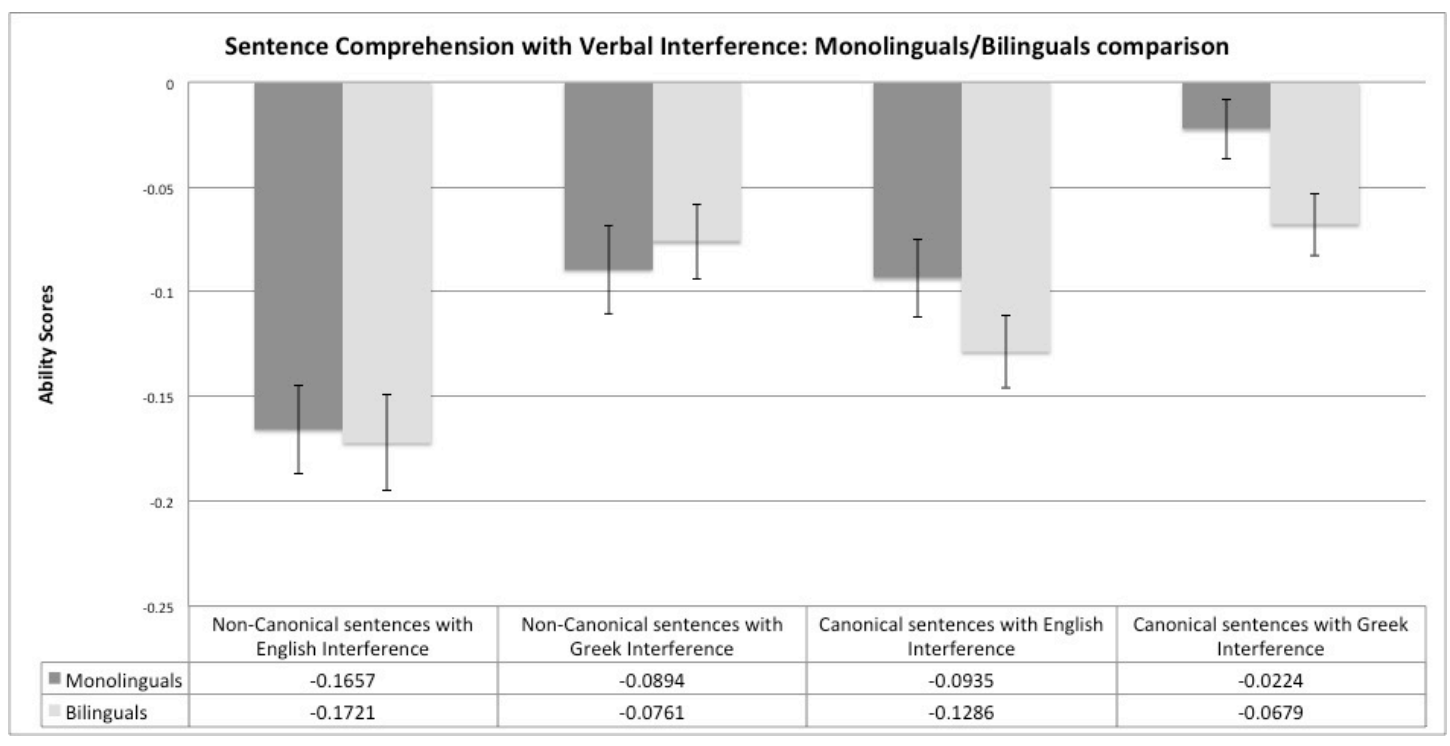

Figure 2: Monolinguals' and bilinguals' ability scores for errors with SE bars in the sentence interpretation task for both canonical and non-canonical sentences in the presence of English and Greek interference.

In summary, behavioural analysis for errors did not reveal any significant difference in comprehension of English target sentences in the presence of English or Greek interference.

\section{Voxel-based morphometry results}

We identified a priori volumes of interest within the cerebellum. We took the peak coordinate reported in Filippi et al. (2011) where there was a positive correlation between grey matter density and control of interference in the sentence interpretation task, and applied a $10 \mathrm{~mm}$ radius volume of interest at that coordinate $(\mathrm{x}=+12 \mathrm{y}=-64 \mathrm{z}=-42)$. For completeness we ran analyses in both hemispheres. All reported effects were significant at $\mathrm{p}$ $=.001$, FWE corrected for multiple comparisons at $\mathrm{p}=.05$.

A direct group comparison revealed more grey matter volume in bilinguals over monolinguals within our volume of interest in the right hemisphere as shown in Table 1 and Figure 3. No effects were observed in the left hemisphere, or for monolinguals over bilinguals. 
Table 1. Differences in grey matter volume for bilinguals relative to monolinguals.

\begin{tabular}{clccc}
\hline \multicolumn{1}{c}{ Effect } & \multicolumn{1}{c}{ Region } & Peak & Z & $\begin{array}{c}\text { P } \\
\text { (FWE } \\
\text { corr) }\end{array}$ \\
\hline Bilinguals > monolinguals & $\begin{array}{l}\text { Right hemisphere: } \\
\text { 10mm VOI at } x+12 y-64 z-42\end{array}$ & $18-63-48$ & 3.5 & .009 \\
& $\begin{array}{l}\text { Left hemisphere: } \\
\mathbf{1 0 m m} \text { VOI at } x-12 y-64 z-42\end{array}$ & - & - & - \\
\hline
\end{tabular}

Note: No effects were observed for monolinguals over bilinguals

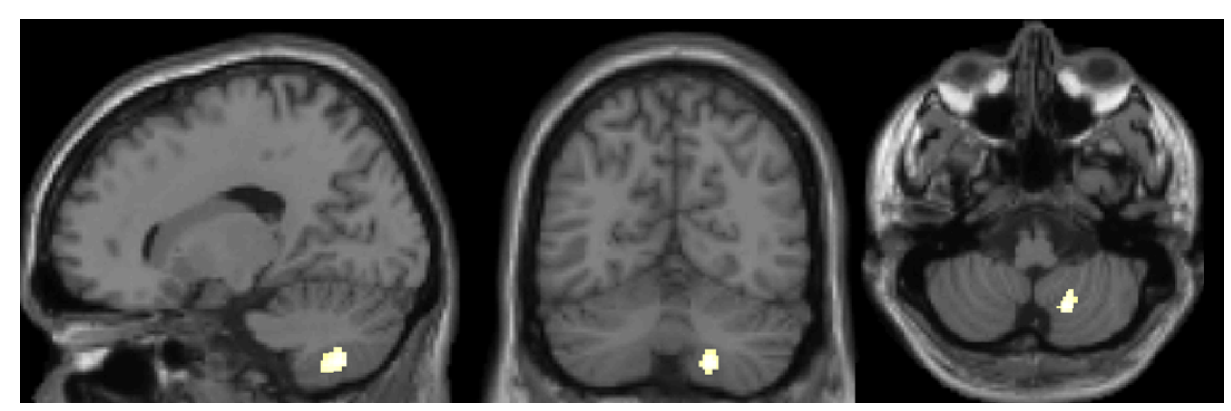

Figure 3. Group comparison of cerebellar grey matter volume, greater in bilinguals.

Note: sections taken at $\mathrm{x}=18 \mathrm{y}=-63 \mathrm{z}=-48$. There were no effects for monolinguals $>$ bilinguals

Volumetric sensitivity to performance on the sentence interference task

There was no main effect for interference (averaged across English and Greek conditions) relative to control trials or sentence type (canonical vs noncanonical) within our volumes of interest. Furthermore, there was no evidence for differences in volumetric sensitivity to the control of English (i.e., familiar) interference relative to control performance within our full sample, within either group or by sentence type (canonical/noncanonical). There was no main effect for control of Greek interference across all participants. However, when we modelled monolingual and bilingual performance on noncanonical sentences separately we found significant volumetric sensitivity in our bilinguals but not our monolinguals and there was a significant group (bilingual/monolingual) x condition (Greek interference/control) interaction. These effects held in both hemispheres, as shown in Table 2 and Figure 4. 
Table 2. Volumetric senstitivity within the cerebellum to performance on the sentence interpretation task.

\begin{tabular}{|c|c|c|c|c|}
\hline Effect & Region & Peak & $\mathbf{Z}$ & $\begin{array}{c}\text { P } \\
\text { (FWE } \\
\text { corr) }\end{array}$ \\
\hline Condition: & $\begin{array}{l}\text { Right hemisphere: } \\
10 \mathrm{~mm} \text { VOI at } x+12 y-64 z-\end{array}$ & & & \\
\hline Greek interference vs control: & 42 & - & - & - \\
\hline $\begin{aligned} \text { Group: } & \text { Bilinguals } \\
& \text { Monolinguals }\end{aligned}$ & & $\begin{array}{c}9-62-51 \\
-\end{array}$ & $\begin{array}{c}3.40 \\
-\end{array}$ & $\begin{array}{c}.012 \\
-\end{array}$ \\
\hline Group $x$ condition interaction & & $9-63-51$ & 3.87 & .003 \\
\hline Condition: & Left hemisphere: & & & \\
\hline Greek interference vs control: & $10 \mathrm{~mm}$ VOI at $x-12 y-64 z-42$ & - & - & - \\
\hline Group: Bilinguals & & $-15-65-48$ & 3.47 & .010 \\
\hline Monolinguals & & - & - & - \\
\hline Group $x$ condition interaction & & $-14-65-50$ & 4.74 & $<.001$ \\
\hline
\end{tabular}

Note. There were no group differences in grey matter sensitivity to scores in the context of English interference and no areas of the cerebellum that showed greater sensitivity to task performance in monolinguals relative to bilinguals.

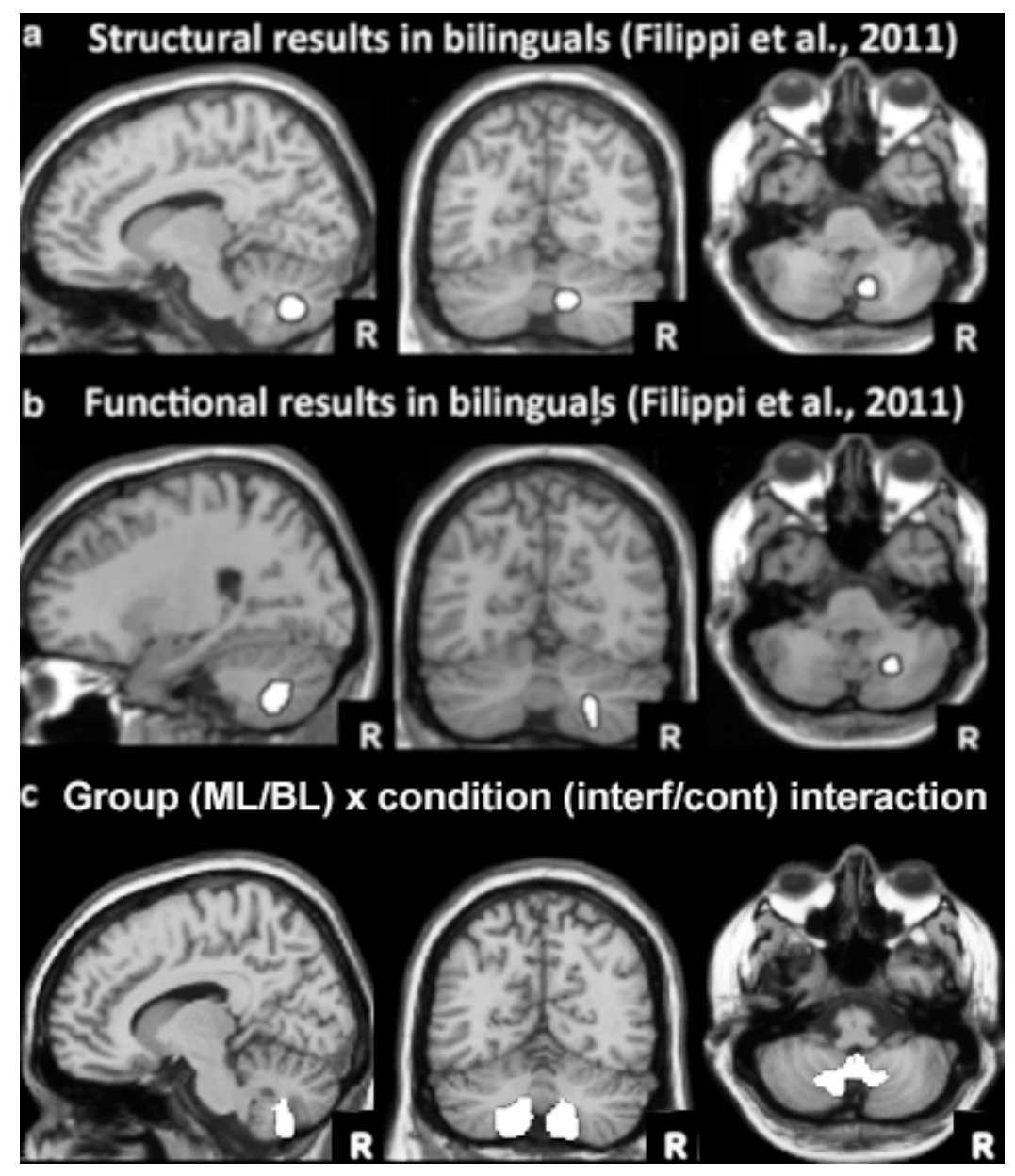

Figure 4. a) Structural imaging results from Filippi et al. (2011) shown at $\mathrm{x}=12, \mathrm{y}=-64, \mathrm{z}=$ 42. White area shows where grey matter density was higher in those bilinguals $(\mathrm{N}=26)$ with better control of interference; b) Functional imaging results from Filippi et al. (2011) shown at $\mathrm{x}=20, \mathrm{y}=$ $64, \mathrm{z}=-42$. White area shows where activation was higher when German participants $(\mathrm{N}=8)$ performed semantic decisions on written words in native vs English interference; c. Results from the current study shown at $\mathrm{x}=12, \mathrm{y}=-62, \mathrm{z}=-45$. White area shows where the difference in 
volumetric sensitivity across conditions (Greek interference and control trials) differed significantly as a function of group (bilingual/monolingual).

The source of the group $\mathrm{x}$ condition interaction, in both the right and left VOI is demonstrated in Figure 5. Here, raw beta values (i.e., unadjusted for the other covariates in our model) are plotted against ability scores (interference minus control performance) on the noncanonical sentences. There was a significant positive correlation between ability score and grey matter volume in our bilingual participants, but this correlation was nonsignificant (and negative) in our monolinguals, a pattern which held true in both hemispheres. In both VOIs, using the Fisher r-to-z transformation, correlation coefficients were significantly stronger in the bilingual sample (LH: $\mathrm{z}=3.19, \mathrm{p}<.01 ; \mathrm{RH}: \mathrm{z}=2.78, \mathrm{p}<.01$, two-tailed).

\section{Grey matter volume and control of Greek interference}

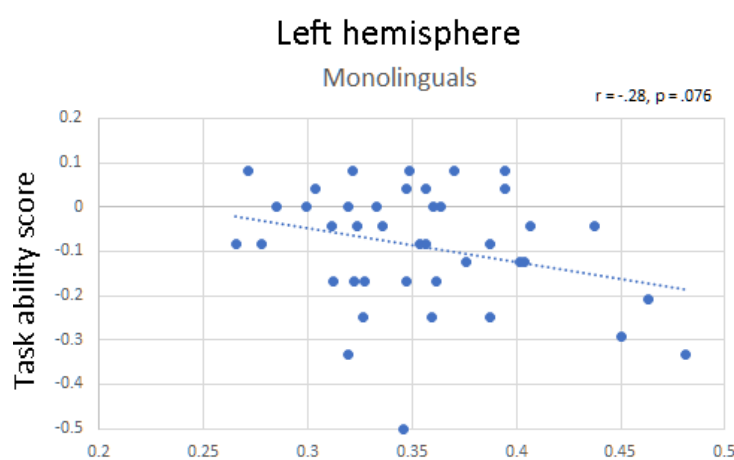

Right hemisphere
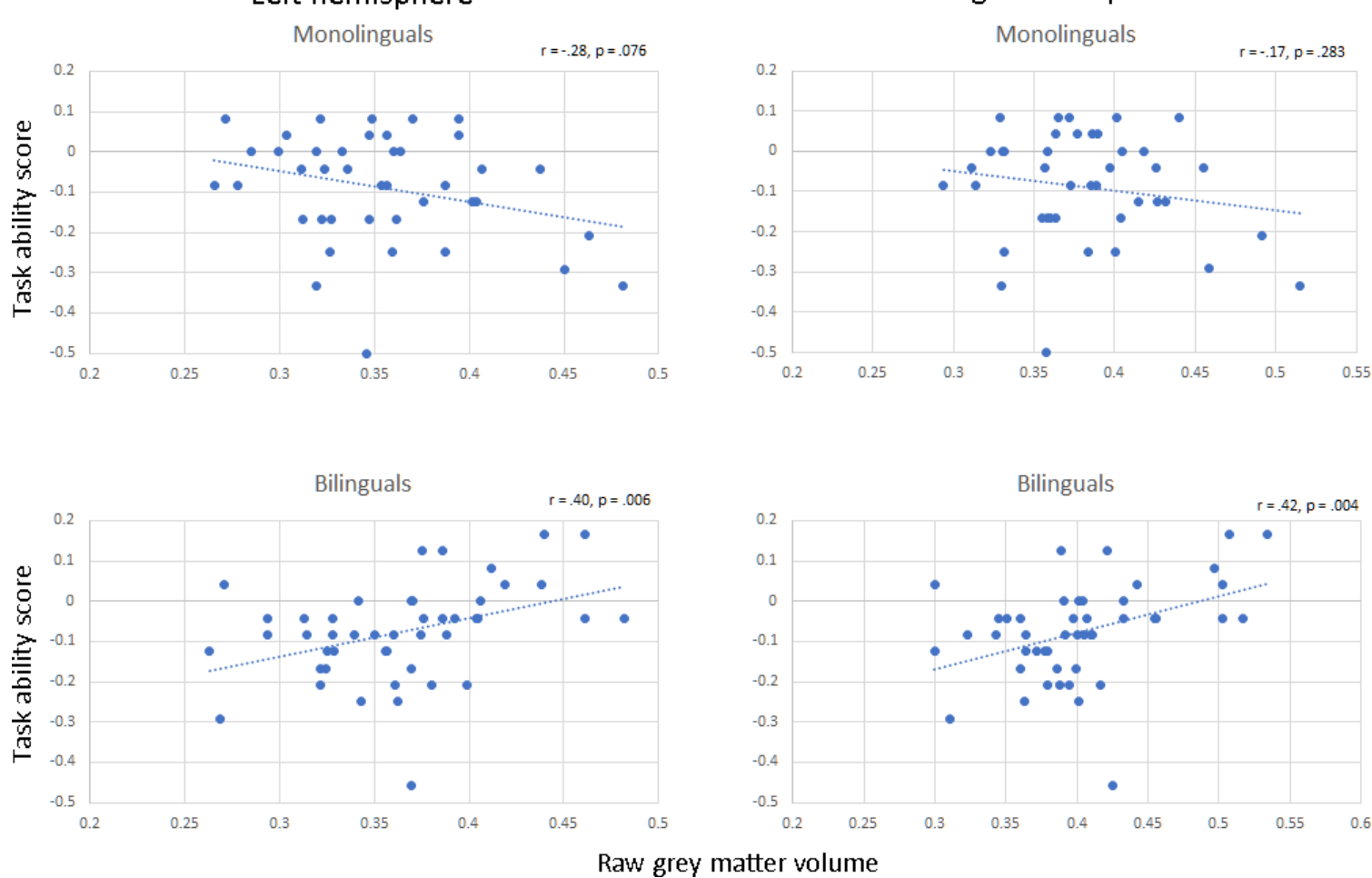

Figure 5. Plots show correlations of raw beta values (grey matter volume) within $10 \mathrm{~mm}$ radius spherical ROIs (centred around $-12-64-42$ and $+12-64-42$ ) against task ability score in the context of Greek interference. 


\section{Discussion}

In this study, we investigated the role of cerebellar areas in the control of interference in speech comprehension. We extended our previous research (which employed only a bilingual sample) to include a sample of English monolinguals.

We acquired raw structural images from a large group of adult individuals $(\mathrm{N}=87)$, split in two groups: English monolinguals $(\mathrm{N}=41)$ and bilinguals of different linguistic backgrounds $(\mathrm{N}=46)$. Their age ranged from 18 to 80 years old. Voxel-based morphometry was used to identify grey matter markers of processing ability on brain structure across the lifespan. Consistent with previous work (Filippi et al., 2011) we first acquired data on a behavioural test and then acquired structural MRI data.

As a result, all participants performed a sentence interpretation task with different levels of grammatical complexity (canonical and non-canonical sentences), in the presence of verbal interference outside the scanner. Verbal interference was generated by the simultaneous presentation of competing speech in English (the language that all participants could speak) and Greek (a language unknown to all of them). Results demonstrated greater absolute volume and volumetric GM sensitivity to Greek interference in BLs relative to MLs in the same area of lobule VIII reported by Filippi et al. (2011).

\section{In what ways does this study differ from our previous findings?}

Our findings support the claim (Filippi et al., 2011) that the cerebellum is functionally involved in controlling verbal interference. Unlike the earlier study, however, the task sensitivity effects were bilateral, perhaps indicating shared/interhemispheric functionality rather than strong lateralisation for language processing and control in the cerebellum. Moreover, in the current study the structure-performance correlations were observed in the modulated rather than unmodulated data, indicating volumetric rather than density sensitivity 
in the cerebellum.

Our data support the claim that the acquisition of a second language impacts on the distributed networks underpinning language processing, but also that these effects may not be a marker for better performance. Thus, while we have detected robust differences in volumetric sensitivity within the cerebellum in the control of verbal interference, as well as greater absolute cerebellar volume in bilinguals, there was no cognitive advantage at the behavioural level. This non-straightforward relationship between structural variability and performance/ability is a theme also highlighted by other authors (Paap \& Greenberg, 2013; Paap, Johnson \& Sawi, 2015; Dunabeitia et al., 2014, Dunabeitia \& Carreiras, 2015; Hiltchey \& Klein, 2011; Klein, 2015).

\section{Why did we find the effect in the control of unfamiliar (Greek) linguistic interference?}

We expected that structure/performance correlations would be strongest when controlling interference in the familiar target language (English sentences with English interference). Contrary to our predictions the effect was reliable only in the unfamiliar language interference condition (English sentences with Greek interference). This unexpected finding suggests that the role of the cerebellum might relate more to the orienting of attention than interference control per se. Although speculative, we suggest that bilinguals may show more sensitivity to unfamiliar languages and therefore develop resources to manage the additional processing demands associated with selectively attending to a known target language against unfamiliar linguistic noise. Therefore, the bilateral volumetric sensitivity in the cerebellum may reflect this requirement to selectively attend to the target language in the control of unfamiliar verbal information.

\section{Summary}

Traditionally, the cerebellum has not been included in models of language processing. More recent research indicates an important role in the cerebellum in language comprehension 
(Moberget and Ivry, 2016) language perception (Schwartze and Kotz, 2016) and language production (Pleger and Timmann, 2018). Our paper contributes to this literature, demonstrating that bilingualism confers structural differences within lobule VIII and may play an important role in the resolution of linguistic competition in noisy environments. Further work should explore the role of the cerebellum in selective attention in language processing at a functional level. Research should also exploit structural MRI to resolve the comparative significance of volumetric and density sensitivity within the cerebellum in the control of linguistic interference. 


\section{References}

Abbate MS, LaChapelle NB (1984) Pictures, please! Tucson (AZ): Communication Skill Builders.

Alexander MP, Stuss DT, Picton T, Shallice T, Gillingham S (2007) Regional frontal injuries cause distinct impairments in cognitive control. Neurology 68:1515-1523.

Ali N, Green DW, Kherif F, Devlin JT, Price CJ (2010) The role of the left head of caudate in suppressing irrelevant words. J Cogn Neurosci 22:2369 -2386.

Dunabeitia JA, Carreiras M (2015) The bilingual advantage: Acta est fabulosa. Cortex 73:371-372.

Dunabeitia JA, Hernandez JA, Anton E, Macizo P, Estevez A, Fuentes LJ, Carreiras M (2014) The inhibitory advantage in bilingual children revisited myth or reality? Exp Psychol $61: 234-251$.

Dunn M, Dunn LM (1997) Peabody Picture Vocabulary Test: Circle Pines (3rd ed.). Minneapolis, MN: AGS.

Filippi R, Richardson FM, Dick F, Leech R, Green DW, Thomas MSC, Price CJ (2011) The Right Posterior Paravermis and the Control of Language Interference. J Cogn Neurosci 31:10732-10740.

Filippi R, Leech R, Thomas MS, Green, DW, Dick F (2012) A bilingual advantage in controlling language interference during sentence comprehension. Biling-Lang Cogn 15: $858-872$.

Filippi R, Morris J, Richardson FM, Bright P, Thomas MS, Karmiloff-Smith A, Marian V (2015) Bilingual children show an advantage in controlling verbal interference during spoken language comprehension. Biling-Lang Cogn 18: 490-501.

Hilchey MD, Klein RM (2011) Are there bilingual advantages on non-linguistic interference 
tasks? Implications for the plasticity of executive control processes. Psychon Bull Rev $18: 625-658$.

Hodge SM, Makris N, Kennedy DN, Caviness VS Jr, Howard J, McGrath L, Steele S, Frazier JA, Tager-Flusberg H, Harris GJ (2010) Cerebellum, language, and cognition in autism and specific language impairment. J Autism Dev Disord 40:300 -316.

Jansen A, Flöel A, Van Randenborgh J, Konrad C, Rotte M, Fo“rster AF, Deppe M, Knecht S (2005) Crossed cerebro-cerebellar language dominance. Hum Brain Mapp 24:165-172.

Klein RM (2015) Is there a benefit of bilingualism of executive functioning? Biling-Lang Cogn 18:29-31.

Krienen FM, Bucker RL (2009) Segregated fronto-cerebellar circuits revealed by intrinsic functional connectivity. Cereb Cortex 19:2485-2497.

Moberget T, Ivry RB (2016) Cerebellar contributions to motor control and language comprehension: Searching for common computational principles. Ann N Y Acad Sci 1369: 154-171.

Paap KR, Greenberg ZI (2013) There is no coherent evidence for a bilingual advantage in executive processing. Cogn Psychol 66:232-258.

Paap KR, Johnson HA, Sawi O (2015) Bilingual advantages in executive functioning either do not exist or are restricted to very specific an undetermined circumstances. Cortex 68:265278.

Pleger B, Timmann D (2018) The role of the human cerebellum in linguistic prediction, word generation and verbal working memory: Evidence for brain imaging, non-invasive cerebellar stimulation and lesion studies. Neuropsychologia 115:204-210.

Raven J, Raven JC, Court JH (1998) Manual for Raven's Progressive Matrices and Vocabulary scales. Section 1: General overview. Oxford, UK: Oxford Psychologists Press. Roland D, Dick F, Elman JL (2007) Frequency of basic English grammatical structures: A 
corpus analysis. J Mem Lang 57:348-379.

Rodriguez-Fornells A, Rotte M, Heinze HJ, No“sselt T, Münte TF (2002) Brain potential and functional MRI evidence for how to handle two languages with one brain. Nature 415:10261029.

Rodriguez-Fornells A, van der Lugt A, Rotte M, Britti B, Heinze HJ,Münte TF (2005) Second language interferes with word production in fluent bilinguals: brain potential and functional imaging evidence. J Cogn Neurosci 17:422- 433.

Schneider W, Eschman A, Zuccolotto A (2002) E-Prime (version 2.0) [Computer software and manual]. Pittsburgh, PA: Psychology Software Tools.

Schwartz M, Kotz SA (2016) Contributions of cerebellar event-based temporal processing and preparatory function to speech perception. Brain Lang 161:28-32.

Schweizer TA, Alexander MP, Susan Gillingham BA, Cusimano M, Stuss DT (2010) Lateralized cerebellar contributions to word generation: a phonemic and semantic fluency study. Behav Neurol 23:31-37.

Snodgrass J G, Vanderwart M (1980) A standardized set of 260 pictures: Norms for name agreement, image agreement, familiarity, and visual complexity. J Exp Psychol Learn Mem Cogn. 6:174-215.

Stoodley CJ, Schmahmann JD (2009) Functional topography in the human cerebellum: a meta-analysis of neuroimaging studies. Neuroimage 44:489-501.

Tzourio-Mazoyer N, Landeau B, Papathanassiou D, Crivello F, Etard O, Delcroix N, Mazoyer B, Joliot M (2002) Automated anatomical labelling of activations in SPM using a macroscopic anatomical parcellation of the MNI MRI single-subject brain. NeuroImage 15: 273-289.

Wechsler D (2008) Wechsler Adult Intelligence Scale (4th ed.). Houston, TX: Pearson. 